\title{
Mass volume measurement in severe head injury: accuracy and feasibility of two pragmatic methods
} Nino Stocchetti, Marco Croci, Diego Spagnoli, Felicetta Gilardoni, Federico Resta,
Angelo Colombo hypertension. ${ }^{1}$ This is especially relevant after traumatic brain injury, when the development of post-traumatic intracranial masses is the most dangerous complication. ${ }^{23}$

In head injury, the volume of masses has been identified as one of the prognostic factors, ${ }^{4}$ and a CT grading system accepted worldwide incorporates volume measurements. ${ }^{5}$

When a cerebral contusion or an extra-axial collection increases, ample variations in volume are easily detected without meticulous measurement. When subtle changes develop over time, on the other hand, the precision provided by more scientific measurement techniques offers a more valuable basis for clinical decision making.

The calculation of a given area, and the reconstruction of a delimited volume, are quite feasible with modern CT apparatus, especially when a dedicated workstation is part of the machinery. Unfortunately, volume determinations are seldom performed during routine CT readings, although the digital measurement of the lesions is the most accurate system available today. Finally, digital measurement proves impossible or cumbersome when multicentre trials are conducted, and central reading of CT is planned.

There is, therefore, a need for pragmatic methods of volume measurement on the CT printouts, provided that those methods are accurate and feasible.

The ellipsoid method (EM) was developed to calculate the volume of arterovenous malformations. ${ }^{6}$ It is based on the concept that the volume of an ellipsoid is about one half the volume of the parallelepiped into which it is placed. By measuring three diameters of a given lesion in the arterial phase of an angiogram, a parallelepiped is reconstructed, and its volume, divided in half, is close to the real volume of the malformation. By extending this geometrical concept from angiography to CT scan, calculation of space occupying lesions becomes possible (fig $1 \mathrm{~A}$ ).

More recently, the Cavalieri direct estimator (CDE) has been introduced. ${ }^{7}$ It breaks down the lesion on CT into a corresponding number of points, through special grids; the volume of a lesion appearing on the scan is the product of the sum of the points that fall on the lesion, the area associated with each point, and the distance between scan slices (fig $1 \mathrm{~B}$ ). The grid can be constructed by photocopying a template provided in the original article ${ }^{7}$ or by preparing a uniformly spaced point grid (by computer or by hand) to be copied onto an overhead transparency. 

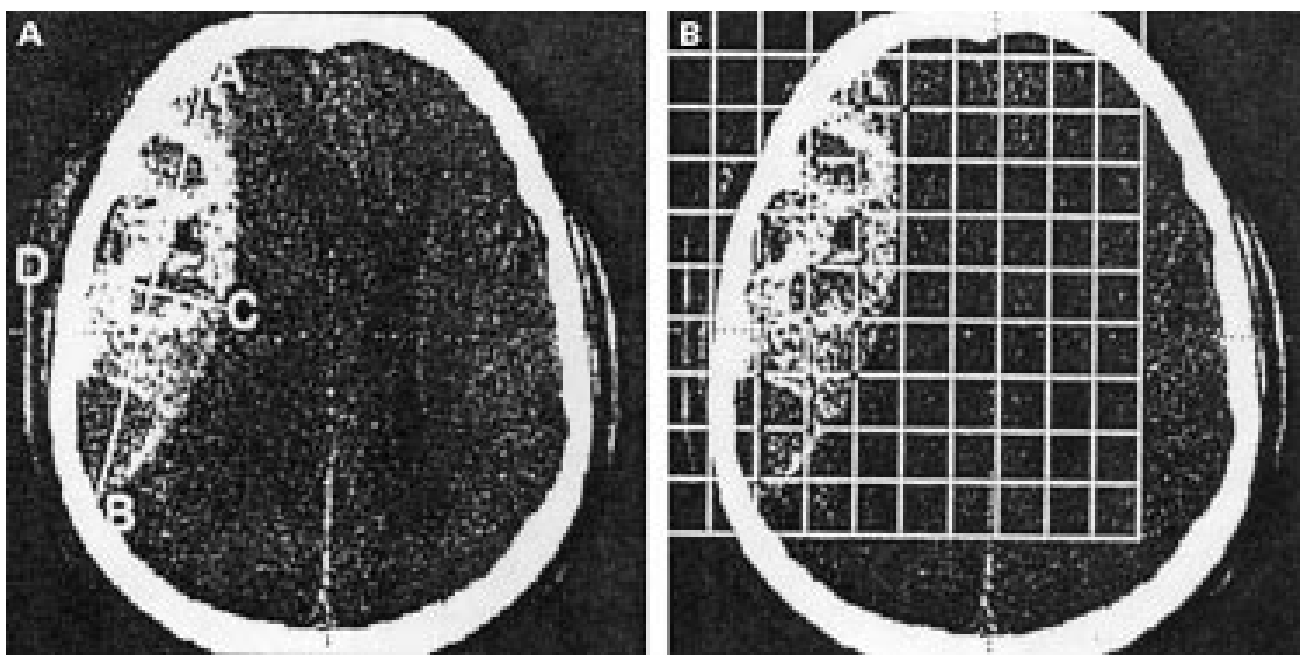

Figure 1 (A)The ellipsoid method is based on the concept that the volume of an ellipsoid is about half the volume of the parallelepiped into which it is placed. By measuring two diameters of a given lesion on a CT slice and considering the number of slices, a parallelepiped is reconstructed, and its volume, divided by half, is close to the real volume of the malformation. (B). The Cavalieri direct estimator breaks down the lesion seen on CT into a corresponding number of points, through special grids; the volume of a lesion appearing on the scan is the product of the sum of the points that fall on the lesion, the area associated with each point, and the distance between scan slices.

As volume measurement is clinically important, but rarely performed in the neuroradiological practice, a posteriori methods, feasible even outside the neuroradiology department, may be useful or necessary. We have therefore assessed the performances and the feasibility of two pragmatic methods (the EM and the CDE) in measuring the volume of mass lesions in cases of patients with severe head injury.

\section{Materials and methods}

Eleven patients with severe head injury admitted to the neuroscience intensive care unit of a university hospital in 1997 were studied. They were eight men and three women, mean age 53 (SD 19) years, with a median Glasgow coma score after resuscitation of 6 . Each patient underwent CT at admission, and repeated controls thereafter. Among the scans of these patients, 19 containing mass lesions were selected. A lesion was defined as a hyperdense or mixed (hyperdense and hypodense) area. Each scan was digitally processed (CT Scan Pace, General Electric, Maryland, USA) to determine the volume of every lesion. Such measurements were taken by a neuroradiologist, who traced every lesion on the screen and calculated the area and volume of interest. That was accepted as the reference gold standard. The results of these measurements were not disclosed to the panel of examiners.

Three members of the intensive care unit staff were trained in the identification and measurement of areas and volumes on the CT slices by using the two pragmatic methods. As part of the training, the examiners performed collective readings of several scans of patients not participating in the study, and repeated measurements of lesions using the CDE and the EM under the guidance of a neuroradiologist. Grids for the CDE were prepared and provided to the examiners. Relevant references in the literature were reviewed. After this training period, which required about 2 weeks, the
19 CT scans and appropriate examination forms were submitted to each panellist separately. The CDE and EM were used in a random order.

Both the volumes measured in each scan and the reading time for each method were recorded in the forms. The data forms were then collected, summarised, and compared with the gold standard.

To assess the agreement between the two pragmatic methods and the gold standard ${ }^{8}$ the statistical method devised by Bland and Altman $^{9}$ was used. A two way analysis of variance (ANOVA) was used for measuring the interobserver variability. The Pearson $\chi^{2}$ test with Yates continuity correction was used for comparing percentages.

\section{Results}

The 19 CT scans studied comprised 23 contusions/lacerations, 10 subdural haematomas, and four extradural haematomas. The range of the lesions directly computed at the CT scan varied from $3 \mathrm{ml}$ to $124 \mathrm{ml}$; the sum of all lesions produced a total volume of $1260.65 \mathrm{ml}$, with an average volume for every lesion of 34.23 (SD 35.88) $\mathrm{ml}$.

READER AGREEMENT AND READINGS

The readings performed by the three examiners led to consistent measurements. The results of their calculations were very close; the mean volume measured by each examiner differed by $<1.5 \mathrm{ml}$. The ANOVA analysis confirmed this finding, excluding any significant difference among the readers in the use of both methods. All 37 lesions were identified by all readers, providing 222 pragmatic measurements to be compared with the gold standard.

The measurements calculated by the three readers with each method for every lesion were averaged; this average represented the volume calculated with the method. The range of the lesions measured with the EM varied from 0.9 


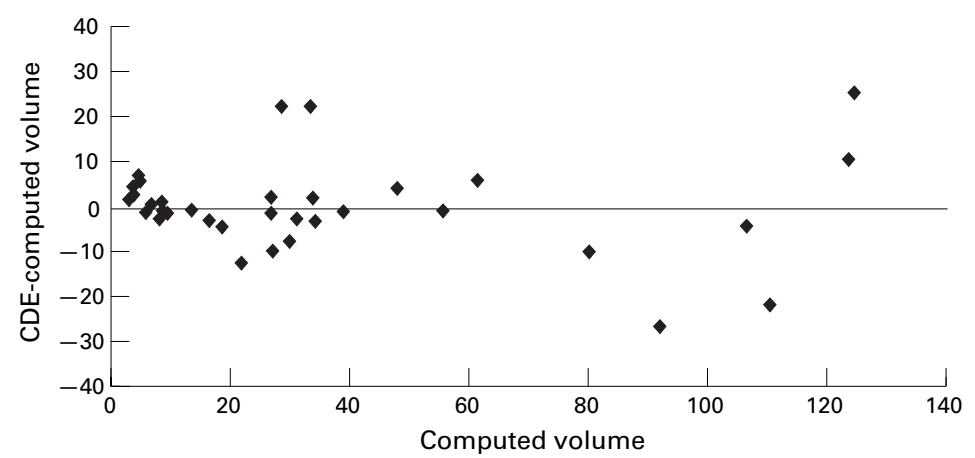

Figure 2 The $y$ axis shows the difference between the volume read with the CDE and the volume measured with the computerised method. This difference is plotted against the $x$ axis, in which the gold standard is reported. The zero line represents the line of equality, corresponding to a perfect agreement between the two methods of measurement. Each point indicates one or more lesions detected on the CT scan. All values are in ml.

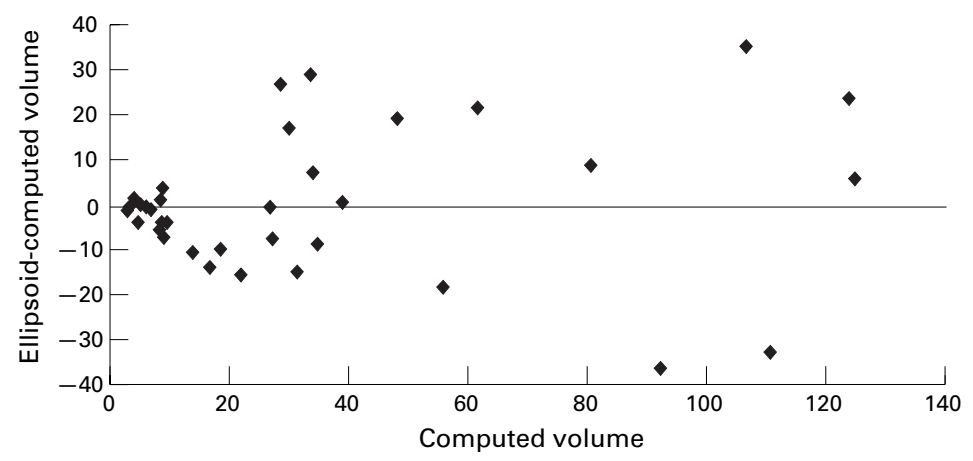

Figure 3 The $y$ axis shows the difference between the volume read with the EM and the volume measured with the computerised method. This difference is plotted against the $x$ axis, in which the gold standard is reported. The zero line represents the line of equality, corresponding to a perfect agreement between the two methods of measurement. Each point indicates one or more lesions detected at the CT scan. All values are in $\mathrm{ml}$.

to $147.4 \mathrm{ml}$; the sum of all lesions produced a total volume of $1273.87 \mathrm{ml}$, with an average volume for every lesion of 34.43 (SD 40.98) $\mathrm{ml}$. The range of the lesions measured with the CDE varied from $4.8 \mathrm{ml}$ to $150 \mathrm{ml}$; the sum of all lesions produced a total volume of 1287.42 $\mathrm{ml}$, with an average volume for every lesion of 34.8 (SD 36.6) $\mathrm{ml}$.

\section{AGREEMENT OF THE TWO METHODS WITH THE} GOLD STANDARD

The agreement between the CDE and EM and the computed reading was acceptable on average, but questionable in some cases.

The average difference between the applied technique and the reference value (termed bias, a measure of systematic error with the statistical method of Bland and Altman ${ }^{9}$ ) was 0.57 (SD 9.99) $\mathrm{ml}$ for the CDE and 0.20 (SD 15.48) $\mathrm{ml}$ for the EM. The $95 \%$ confidence interval $(95 \% \mathrm{CI})$ for this bias fell between

Intracerebral versus extracerebral lesions

\begin{tabular}{lcllll}
\hline & & $\begin{array}{l}\text { Mean } \\
\text { Volume } \\
\text { (SD) }\end{array}$ & $\begin{array}{l}\text { Bias } \\
\text { (SD) }\end{array}$ & $\begin{array}{l}\text { Limits of } \\
\text { agreement }\end{array}$ & $\begin{array}{l}\text { 95\% CI } \\
\text { for the bias }\end{array}$ \\
\hline Gold standard: & No & & & & \\
$\quad$ Intracerebral & 23 & $28.7(30)$ & & & \\
$\quad \begin{array}{l}\text { Extracerebral } \\
\quad 14\end{array}$ & $43.3(43)$ & & & \\
Cavalieri direct estimator: & & & & \\
$\quad$ Intracerebral & 23 & $27.5(25)$ & $-1.91(9.07)$ & -21.4 to +17.6 & -6.0 to +2.21 \\
$\quad$ Extracerebral & 14 & $47.9(48)$ & $4.64(9.2)$ & -13.8 to +23 & -0.7 to +9.97 \\
$\begin{array}{l}\text { Ellipsoid method: } \\
\quad \text { Intracerebral }\end{array}$ & & $26.7(28)$ & $-1.19(14.3)$ & -29.8 to +27.4 & -7.4 to +5 \\
$\quad$ Extracerebral & & $45.8(55)$ & $2.49(17.5)$ & -32.6 to +37.6 & -7.7 to +12.6 \\
\hline
\end{tabular}

-2.75 and $3.89 \mathrm{ml}$ for the $\mathrm{CDE}$, and between -4.94 and $5.35 \mathrm{ml}$ for the EM. The maximum difference between the pragmatic readings and the reference value was in the order of $25 \mathrm{ml}$ with the CDE and reached $35 \mathrm{ml}$ with the EM.

A graphical description of the agreement between each pragmatic method and the gold standard is illustrated in figs 2 and 3 . In these plots the distance between the line of equality, lying on the zero difference between the pragmatic method and the reference reading, expresses the discrepancy between each measurement and the true value. There are discrepancies among the two pragmatic methods and the digital recording in individual cases. With the CDE these discrepancies are few and more evident at large volumes; with the EM the discrepancies are more frequent and present at lower volumes.

The limits of agreement, an additional mathematical descriptor of the agreement, were between -19.41 and $20.55 \mathrm{ml}$ with the CDE and varied between -30.76 and $31.16 \mathrm{ml}$ with the EM.

MASSES GREATER THAN 25 ML

Since a volume greater than $25 \mathrm{ml}$ is considered a threshold for surgical indication, the capability of appropriately identifying this cut off value may indicate the clinical reliability of pragmatic methods. Nineteen lesions $>25$ $\mathrm{ml}$ were found from the CT analysis. Seventeen lesions $>25 \mathrm{ml}$ were identified with the CDE and 16 were detected with the EM. There is not a statistical significant difference between these two findings.

EXTRACEREBRAL AND INTRACEREBRAL LESIONS Volume may become more difficult to measure in lesions of a particular shape. Extracerebral lesions, such as subdural haematomas for example, may be more difficult to measure than a rounded contusion in the brain parenchyma. The performances of the two pragmatic methods in determining the volume of intracerebral or extracerebral lesions were, in fact, different. The CDE was more accurate than the EM, especially in cases of extracerebral masses. The main data are reported in the table.

READING TIME AND LEARNING EFFECT

The average reading time to complete the measurement of a CT scan was 7 minutes 44 seconds (SD 42 seconds) for the CDE method and 4 minutes 27 seconds (SD 38 seconds) for the EM $(\mathrm{p}<0.001)$. As a reference, the average reading time using the digital system was 2 minutes 54 seconds (SD 26 seconds).

In both methods the reading time for each CT was higher at the beginning of this research and decreased as the study proceeded. Such decrease was probably due to a learning effect, as the accuracy of the measurement did not change over time. The examiners became more experienced and read the CT faster, but not at the price of reducing the precision of their analysis. The reduction in reading time was $37 \%$ with the CDE method, and $48 \%$ with the EM. 


\section{Discussion}

The volume of intracranial lesions, and its change over time, is of obvious interest in the diagnosis and management of patients with head injury. Specific features of space occupying lesions, including their volume, are associated with the outcome of patients with traumatic brain injury.

Based on data from the traumatic coma data bank, ${ }^{10}$ Marshall et $a \bar{l}^{\bar{p}}$ showed that the existence of specific findings from CT, including the presence of masses exceeding $25 \mathrm{ml}$, was associated with increasing degrees of mortality and morbidity. This scale is now widely used both in clinical practice and in the scientific literature on head injury. Interestingly, this scale requires the measurement of the volume of intracranial lesions, as the threshold of $25 \mathrm{ml}$ is part of the grading.

In some clinical trials recently performed on head injury, a central reading of the CT scan was carried out, ${ }^{11}{ }^{12}$ and intracranial volume measurements performed. ${ }^{11}$

There are clinical problems, such as the evolution of a cortical contusion, that require volume measurement. The overall mass effect, which is considered for clinical management, depends on the size of a given lesion and on the associated swelling and oedema as well, but the size of the lesion itself is crucial.

For these reasons an accurate estimate or, possibly, the measurement of the volume of cerebral masses has become an integral part of the treatment and of the classification of severe head injury.

Unfortunately, the determination of the volume of masses is not performed as an element of the routine reading of CT scans. In a recent multicentre study on head injury ${ }^{13}$ involving 18 neurotraumatological centres, all 18 centres claimed to have used the Marshall scale, but the volume of lesions was seldom measured in one centre, and never measured in the other 17 (personal unpublished data).

The data collected in this study show that both pragmatic methods are on average acceptably precise when performed by readers who underwent a short training period. Accuracy was achieved by all readers, as no difference was found among them in their calculation of the volume. The systematic error of the pragmatic methods was $<1 \mathrm{ml}$, and the mean volume of all lesions measured with the $\mathrm{EM}$ and CDE was very close to the volume measured digitally. When considering individual lesions rather than the average volume, however, discrepancies were detected with both methods and some discrepancies were relevant especially with the EM.

The CDE is more accurate than the EM. That is proved by the Bland and Altman analysis, as suggested by the comparison of figs 2 and 3. The bias is lower with the EM because this method determines both overestimates and underestimates, leading to a narrow final mean value. By comparing statistical measures of dispersion, such as SD, or specific parameters of the Bland and Altman analysis, such as the limits of agreement, it seems that the CDE provides measurements closer to the reference value.

Similarly, this method performed better in cases of extracranial lesions.

The EM was developed to assess the volume of vascular malformations and it is not surprising that it does not excel in the head injury field. The EM shows its weakness mainly when extracerebral collections are concerned. In fact, the shape of these lesions is less suitable for calculation by the ellipsoid theory. By contrast, the CDE method may accurately take into account even the irregular shape of thin lesions.

Such greater accuracy of the CDE technique is counterbalanced by the need for a longer execution time; this system takes twice as long to perform as the EM. The necessary reading time, however, is initially 8 minutes but, after a brief training, it decreases to an average of 5 minutes 11 seconds for each CT. With this reduction due to practice, the reading time of each scan with the CDE remains fully acceptable in the clinical setting.

Both pragmatic methods are inferior to computer based reading, which is the choice when accurate volume estimation is necessary. However, if a digital volumetric determination of the lesions using a CT computer is not possible, the two pragmatic methods offer an alternative.

They may be mastered after short training, do not require expensive equipment, and provide a different level of accuracy. The CDE performs better than the EM but takes longer. It is recommended whenever accuracy of measurement is crucial for possible therapeutic decisions.

1 Marmarou A, Tabaddor K. Intracranial pressure: physiology and pathophysiology. In: Cooper PR, ed. Head injury. Baltimore: Williams and Wilkins, 1987:159-176.

2 Bullock R, Teasdale G. Surgical management of traumatic intracranial haematomas. In: Vinken PJ, Bruyn GW, intracranial haematomas. In: Vinken PJ, Bruyn GW, Klawans HJ, et al, eds. Handbook of clinic

3 Chiles BW, Cooper PR. Extra-axial hematomas. In: Loftus $\mathrm{CM}$, ed. Neurosurgical emergencies. Vol I. American Association of Neurological Surgeons: 1994:73-100.

4 Gennarelli TA, Spielman GM, Langfitt TW, et al. Influence of the type of intracranial lesion on outcome from severe head injury. $\mathcal{F}$ Neurosurg 1992;56:26-32.

5 Marshall LF, Marshall SB, Klauber MR, et al. A new classification of head injury based on computerized tomography. 7 Neurosurg 1991;75:s 17-20.

6 Pasqualin A, Barone G, et al. The relevance of anatomic and hemodynamic factors to a classification of cerebral arterovenous malformations. Neurosurgery 1991;28:370-9.

7 Clatterbuck RE, Sipos EP. The efficient calculation of neurosurgically relevant volumes from computed tomographic scans using Cavalieri's direct estimator. Neurosurgery 1997; 10:339-43.

8 LaManthia KR, O'Connor T, Barash PG. Comparing methods of measurement: an alternative approach. Anesthemethods of measureme

9 Bland JM, Altman DG. Statistical methods for assessing agreement between two methods of clinical measurement. Lancet 1986;8:307-10

10 Foulkes MA, Eisenberg HM, Jane JA, et al. The traumatic coma data bank: design, methods, and baseline characteristics. F Neurosurg 1991;75:s8-13.

11 Marshall LF, Maas AI, Marshall SB, et al. A multicenter trial on the efficacy of using tirilazad mesylate in cases of head injury. F Neurosurg 1998;89:519-25.

12 Harders, A, Kakarieka A, Braakman R, et al. Traumatic subarachnoid hemorrhage and its treatment with nimodipine. 7 Neurosurg 1996;85:82-9.

13 Citerio G, Cormio M, Beretta L, et al. A multi-centre Italian head injury database. The neurolink project. Anesthesia 2000 1998;1:81. 